\title{
Symmetry coefficients and incompressibility of clusterized supernova matter
}

\author{
S. K. Samaddar, ${ }^{1, *}$ J. N. De, ${ }^{1, \dagger}$ X. Viñas, ${ }^{2, \ddagger}$ and M. Centelles ${ }^{2, \S}$ \\ ${ }^{1}$ Saha Institute of Nuclear Physics, 1/AF Bidhannagar, Kolkata 700 064, India \\ ${ }^{2}$ Departament d'Estructura i Constituents de la Matèria, Facultat de Física, and Institut de Ciències del Cosmos, \\ Universitat de Barcelona, Diagonal 647, E-08028 Barcelona, Spain
}

(Received 26 June 2009; published 14 September 2009)

\begin{abstract}
The symmetry energy coefficients, incompressibility, and single-particle and isovector potentials of clusterized dilute nuclear matter are calculated at different temperatures employing the $S$-matrix approach to the evaluation of the equation of state. Calculations have been extended to understand the aforesaid properties of homogeneous and clusterized supernova matter in the subnuclear density region. A comparison of the results in the $S$-matrix and mean-field approach reveals some subtle differences in the density and temperature region we explore.
\end{abstract}

DOI: $10.1103 /$ PhysRevC.80.035803

PACS number(s): 21.65.Mn, 21.65.Ef, 24.10.Pa, 26.50.+x

\section{INTRODUCTION}

Studies of the dynamical evolution of supernovae require an accurate knowledge of the properties of nuclear matter over a large range of densities $\rho$, temperatures $T$, and proton concentrations $Y_{p}$ [1,2]. The density values range from a few times the normal nuclear matter density $\rho_{0}$ to $\sim 10^{-7} \rho_{0}$, the temperature may be as high as $\sim 20 \mathrm{MeV}$, and the proton concentration may even be close to zero. Based on the laboratory experiments, the properties of cold $(T=0)$ nuclear matter around the saturation density $\rho_{0}$ with proton concentration limited by the stability valley of finite nuclei are more or less well known. Outside this narrow limit, the understanding of the properties of nuclear matter is fraught with many uncertainties.

The density dependence of the nuclear incompressibility and of the symmetry energy are among the key elements in the simulation dynamics of supernova explosion [3]. At supranormal densities, nuclear flow in energetic nuclear collisions aided by model-dependent calculations helps to explain the nuclear incompressibility [4-6]. Similarly, inference can be made on the density dependence of the symmetry energy from the comparison of theoretical predictions with experimental data on the differential flow of neutrons and protons and from the $\pi^{-} / \pi^{+}, K^{0} / K^{+}$ratios, etc. [7-9]. Disassembly of hot expanded nuclei offers one of the best tools to study the characteristics of the nuclear symmetry energy at subnormal densities [10-12]. Experimental data related to isotopic distributions [13], isospin diffusion [14-16], and isoscaling $[10,17]$ constrain the density dependence of the symmetry energy in the subnormal region. There is considerable uncertainty, however, in all these extractions. The importance of the nuclear symmetry energy can be gauged by the fact that higher symmetry energy, for example, leads to a lower electron $\left(e^{-}\right)$-capture rate in the supernova collapse phase that may result in a strong explosive shock $[1,18]$. The

\footnotetext{
*santosh.samaddar@saha.ac.in

†jn.de@saha.ac.in

${ }^{\ddagger}$ xavier@ecm.ub.es

§mariocentelles@ub.edu
}

isotopic abundance of relatively heavier elements in explosive nucleosynthesis is further directly correlated to the symmetry energy.

Microscopic approaches based on realistic $N N$ interactions, Brueckner or variational schemes, or on effective field theories show a large range of predictions on the density dependence of the nuclear incompressibility [19-24] and symmetry energy [7,25-29]. These results are model dependent, which seems unavoidable at near-normal densities and above. At lower densities, however, based on the general analysis of the grand-canonical partition function for nuclear matter in the $S$-matrix framework [30,31], it is possible to have predictions for various nuclear observables which are practically model independent. For dilute nuclear matter, the system minimizes its total free energy by forming clusters. The observables are expressed in terms of specific known properties of these cluster species like their binding energies, scattering phase shifts, etc. Using the virial expansion technique, Horowitz and Schwenk [32] have evaluated the symmetry energy coefficients of clusterized dilute nuclear matter where the cluster species were neutrons, protons, and $\alpha$ particles. In the $S$-matrix approach, the calculations in nuclear matter were extended with inclusion of all possible heavier clusters [30]. The so-calculated results were found to be appreciably different from those obtained in Ref. [32].

In charge-free nuclear matter, the fragment species, in principle, may be infinite in size. In supernova matter (chargeneutral due to the presence of electrons), the Coulombic term in the binding energies of the fragments severely constrains their size within the limits of the drip lines. The fragment composition is then likely to be altered, which would affect the properties of supernova matter in contrast to those in nuclear matter. The aim of the present paper is to investigate the symmetry and compression properties of supernova matter in the low-density regime (up to $\sim 0.02 \mathrm{fm}^{-3}$ ) along with those for nuclear matter. Specifically, the density and temperature dependence of the symmetry energy coefficients, the isovector potentials, and the symmetry incompressibilities are explored.

The paper is organized as follows. The theoretical details are presented in Sec. II. The results and discussions are contained in Sec. III, and the concluding remarks are given in Sec. IV. 


\section{ELEMENTS OF THEORY}

The logical framework of the theory is set in the grandcanonical partition function of the interacting quantum system in the $S$-matrix formalism of statistical mechanics as proposed by Dashen, Ma, and Bernstein [33]. In Sec. II A, we present the key elements of the theory [30] as applied to nuclear matter or supernova matter. In Sec. II B, the expressions for a few relevant observables are given. In Sec. II C we present the methodology for calculating the single-particle and isovector potentials and the symmetry coefficients in some detail.

\section{A. The $S$-matrix framework}

The grand-canonical partition function for the twocomponent nuclear matter composed of neutrons and protons as the elementary species is written as

$$
\mathcal{Z}=\operatorname{Tr} e^{-\beta\left(H-\mu_{p} \hat{N}_{p}-\mu_{n} \hat{N}_{n}\right)},
$$

where $\beta$ is the inverse of the temperature $T$ of the system, $H$ the total Hamiltonian, $\hat{N}_{p, n}$ the number operators for protons and neutrons, and $\mu_{p, n}$ are the corresponding chemical potentials. The trace is taken over any complete set of states of all possible number of nucleons. Denoting the elementary fugacities by $\zeta_{p}=e^{\beta \mu_{p}}$ and $\zeta_{n}=e^{\beta \mu_{n}}$, the full trace can be decomposed as

$$
\mathcal{Z}=\sum_{Z, N=0}^{\infty}\left(\zeta_{p}\right)^{Z}\left(\zeta_{n}\right)^{N} \operatorname{Tr}_{Z, N} e^{-\beta H}
$$

where $\operatorname{Tr}_{Z, N}$ is taken over states of $Z$ protons and $N$ neutrons. For small $\zeta_{p}$ and $\zeta_{n}$, the quantity $\ln \mathcal{Z}$ can be expanded in a virial series

$$
\ln \mathcal{Z}=\sum_{Z, N}^{\prime} D_{Z, N}\left(\zeta_{p}\right)^{Z}\left(\zeta_{n}\right)^{N}
$$

Here the prime indicates that the term with $Z=N=0$ is excluded. The knowledge of the virial coefficients $D_{Z, N}$ gives the partition function and thence the thermodynamic observables.

In Ref. [33], it was shown that all the dynamical information concerning the microscopic interaction in the grand potential of the system is contained in the partition function as two types of terms:

$$
\ln \mathcal{Z}=\ln \mathcal{Z}_{\text {part }}^{(0)}+\ln \mathcal{Z}_{\text {scat }},
$$

corresponding to contributions from stable single-particle states of clusters of different sizes (neutrons and protons included) formed in the infinite system and (multiparticle) scattering states, respectively. The superscript (0) indicates that the clusters behave like an ideal quantum gas. The first term can further be decomposed into contributions from ground states and excited states below nucleon emission threshold. Then,

$$
\ln \mathcal{Z}_{\text {part }}^{(0)}=\ln \mathcal{Z}_{\mathrm{gr}}^{(0)}+\ln \mathcal{Z}_{\mathrm{ex}}^{(0)},
$$

with

$$
\ln \mathcal{Z}_{\mathrm{gr}}^{(0)}=\mp V \sum_{Z, N} g_{0} \int \frac{d \mathbf{p}}{(2 \pi)^{3}} \ln \left(1 \mp \zeta_{Z, N} e^{-\beta\left(p^{2} / 2 A m\right)}\right) .
$$

The upper and lower signs in the above equation correspond to bosons and fermions, respectively, with $g_{0}$ as the ground-state spin degeneracy. For mass numbers $A \leqslant 8$, the $g_{0}$ values are taken from experiment. For heavier nuclei, $g_{0}$ is taken as 1 or 2 depending on whether the nuclei are bosonic or fermionic. The sum in Eq. (6) extends over all possible fragment species that can be formed; $Z, N$, and $A$ refer to the proton, neutron, and mass number of a species, respectively. Here, $\mathbf{p}$ refers to the momentum of the fragment, $m$ is the nucleon mass, and $V$ is the volume of the system. The effective fugacity is given by $\zeta_{Z, N}=e^{\beta\left(\mu_{Z, N}+B_{Z, N}\right)}$, where the chemical potential of the fragment is $\mu_{Z, N}=Z \mu_{p}+N \mu_{n}$ from the conditions of chemical equilibrium. The quantity $B_{Z, N}$ represents the binding energy of the fragment.

In nuclear matter, only nuclear forces contribute to the binding energy as the Coulomb interaction is absent; then the fragment size can, in principle, be infinite. In supernova matter, the Coulomb interaction is operative in determining the binding energy and the size of the nuclei; the sum in Eq. (6) is then finite. The nuclei are embedded in a sea of electrons and the binding energy gets dressed up; the correction can be estimated using the Wigner-Seitz approximation [34]. We work in natural units $\hbar=c=1$. The integral in Eq. (6) can be expanded in powers of the effective fugacity $\zeta_{Z, N}$ as

$$
\ln \mathcal{Z}_{\mathrm{gr}}^{(0)}=V \sum_{Z, N} \frac{g_{0}}{\lambda_{A}^{3}}\left(\zeta_{Z, N} \pm \frac{\zeta_{Z, N}^{2}}{2^{5 / 2}}+\cdots\right)
$$

The quantity $\lambda_{A}=\sqrt{2 \pi /(A m T)}$ is the thermal wavelength of a species of mass $A m$.

A nucleus in a particular excited state is taken as a distinctly different species and can be treated in the same footing as the ground state. The density of states is quite high in relatively heavy nuclei and increases nearly exponentially with the square root of the excitation energy $E^{*}$. Thus, the contribution of the excited states of a single nucleus is given as an integral over $E^{*}$ of the ideal gas terms weighted with the level density $\omega\left(A, E^{*}\right)$ :

$$
\begin{aligned}
\ln \mathcal{Z}_{\mathrm{ex}}^{(0)}= & \mp V \sum_{Z, N}^{\prime} \int_{E_{0}}^{E_{s}} d E^{*} \omega\left(A, E^{*}\right) \\
& \times \int \frac{d \mathbf{p}}{(2 \pi)^{3}} \ln \left(1 \mp \zeta_{Z, N} e^{-\beta\left(p^{2} / 2 A m+E^{*}\right)}\right) .
\end{aligned}
$$

The expression for the level density is obtained from the Fermi gas model of noninteracting nucleons in a nucleus [35]

$$
\omega(A, E)=\frac{\sqrt{\pi}}{12 a^{1 / 4}} \frac{e^{2 \sqrt{a E}}}{E^{5 / 4}} .
$$

The level density parameter $a$ is taken as $A / 8 \mathrm{MeV}^{-1}$, its empirical value. In Eq. (8), the prime indicates exclusion of light nuclei $(A \leqslant 8)$ in the sum. The lower limit $E_{0}$ is dictated by the location of the first excited state. We take it to be $2 \mathrm{MeV}$. The upper limit $E_{s}$ is the particle emission threshold taken as $8 \mathrm{MeV}$. 
The scattering term in Eq. (4) can be formally written for the system under consideration as

$$
\ln \mathcal{Z}_{\text {scat }}=\sum \int d E \frac{e^{-\beta(E-\mu)}}{2 \pi i} \operatorname{Tr}\left(\mathcal{A} S^{-1}(\epsilon) \frac{\partial}{\partial E} S(\epsilon)\right)_{c},
$$

where the sum is over all scattering channels, each having its chemical potential $\mu$ and formed by taking any number of particles from any of the stable species. The trace is over all the plane wave states for each of the channels. $S$ is the scattering operator and $\mathcal{A}$ is the boson symmetrization or fermion antisymmetrization operator. The subscript $c$, in diagrammatic language, refers to only the connected parts of the expression in parenthesis.

To recast Eq. (10) explicitly in the context of nuclear or supernova matter, a set of channels with total proton number $Z_{t}$, neutron number $N_{t}$, and mass number $A_{t}$ will be considered. All other labels required to fix a channel are denoted by $\sigma$. Obviously, the total mass and the chemical potential are independent of $\sigma$, depending only on $Z_{t}$ and $N_{t}$. The nonrelativistic energy in a channel is given by

$$
E_{Z_{t}, N_{t}, \sigma}=\frac{P_{\mathrm{c.m} .}^{2}}{2 A_{t} m}-B_{Z_{t}, N_{t}, \sigma}+\epsilon
$$

where $\mathbf{P}_{\text {c.m. }}$ is the total center-of-mass momentum, $\epsilon$ is the kinetic energy in the c.m. frame, and $B_{Z_{t}, N_{t}, \sigma}$ is the sum of the individual binding energies of all the fragments in the channel. Integrating over $\mathbf{P}_{\mathrm{c} . \mathrm{m} .}$, one then gets

$$
\begin{aligned}
\ln \mathcal{Z}_{\text {scat }}= & V \sum_{Z_{t}, N_{t}} \frac{e^{\beta \mu_{Z_{t}, N_{t}}}}{\lambda_{A_{t}}^{3}} \sum_{\sigma} e^{\beta B_{Z_{t}, N_{t}, \sigma}} \\
& \times \int_{0}^{\infty} d \epsilon \frac{e^{-\beta \epsilon}}{2 \pi i} \operatorname{Tr}_{Z_{t}, N_{t}, \sigma}\left(\mathcal{A} S^{-1}(\epsilon) \frac{\partial}{\partial \epsilon} S(\epsilon)\right)_{c},
\end{aligned}
$$

the trace being now restricted to the channel $\left(Z_{t}, N_{t}, \sigma\right)$. Examination of Eq. (12) shows that larger binding energies are more important, because of the factor $e^{\beta B_{Z_{t}, N_{t}, \sigma}}$. Furthermore, two-particle channels are expected to be more dominant than the multiparticle channels with the same $Z_{t}$ and $N_{t}$ from binding energy considerations. The two-particle scattering channels are therefore only considered. It becomes convenient to divide the channels into light ones, consisting of low-mass particles $(A \leqslant 8$, say) and heavy ones, containing at least one high-mass particle $(A>8)$, so that we write

$$
\ln \mathcal{Z}_{\text {scat }}=\ln \mathcal{Z}_{\text {scat }}^{L}+\ln \mathcal{Z}_{\text {scat }}^{H} .
$$

The scattering of relatively heavier nuclei is known to be dominated by a multitude of narrow resonances near the continuum threshold. The $S$-matrix elements are then approximated by resonances. Each of these resonances can be treated [36,37] like an ideal gas term. Then, $\ln \mathcal{Z}_{\text {scat }}^{H}$ can be written in the form of $\ln \mathcal{Z}_{\text {ex }}^{(0)}$, assuming the resonance level densities to be the same as those of the excited states given by Eq. (9). The sum of the contributions from the excited and the resonance states can then be written as

$$
\begin{aligned}
\ln \mathcal{Z}_{\mathrm{ex}}^{(0)}+\ln \mathcal{Z}_{\mathrm{scat}}^{H}= & \mp V \sum_{Z, N}^{\prime} \int_{E_{0}}^{E_{r}} d E^{*} \omega\left(A, E^{*}\right) \\
& \times \int \frac{d \mathbf{p}}{(2 \pi)^{3}} \ln \left(1 \mp \zeta_{Z, N} e^{-\beta\left(p^{2} / 2 A m+E^{*}\right)}\right) \\
= & V \sum_{Z, N}^{\prime} \frac{1}{\lambda_{A}^{3}}\left(f_{1} \zeta_{Z, N} \pm f_{2} \frac{\zeta_{Z, N}^{2}}{2^{5 / 2}}+\cdots\right) .
\end{aligned}
$$

The integration in Eq. (14) extends up to $E_{r}$, the limit of resonance domination. The damping of the integral in Eq. (14) due to the presence of the Boltzmann factor limits the contributions to only those from low energies; we take $E_{r}=12 \mathrm{MeV}$. The $A$-dependent entities

$$
f_{n}(A)=\int_{E_{0}}^{E_{r}} d E^{*} \omega\left(A, E^{*}\right) e^{-n \beta E^{*}}
$$

with $n=1,2, \ldots$, decrease steadily with increasing $n$, so that the series converges quite fast. For the evaluation of $\ln \mathcal{Z}_{\text {scat }}^{L}$ (i.e., the contribution of light particles to $\ln \mathcal{Z}_{\text {scat }}$ ), only the scattering channels $N N, N t, N \mathrm{He}^{3}, N \alpha$, and $\alpha \alpha$ are considered, where $N$ and $t$ refer to the nucleon and the triton, respectively. Then,

$$
\ln \mathcal{Z}_{\text {scat }}^{L}=\ln \mathcal{Z}_{N N}+\ln \mathcal{Z}_{N t}+\ln \mathcal{Z}_{N \mathrm{He}^{3}}+\ln \mathcal{Z}_{N \alpha}+\ln \mathcal{Z}_{\alpha \alpha} .
$$

Each of the terms in Eq. (17) can be expanded in the respective virial coefficients. We consider the expansion up to the secondorder coefficients which are written as energy integrals in terms of the relevant phase shifts.

In summary, the grand partition function for the interacting nuclear system is given as

$$
\ln \mathcal{Z}=\ln \mathcal{Z}_{\text {gr }}^{(0)}+\left(\ln \mathcal{Z}_{\text {ex }}^{(0)}+\ln \mathcal{Z}_{\text {scat }}^{H}\right)+\ln \mathcal{Z}_{\text {scat }}^{L} .
$$

Once the partition function is known, the chosen observables can be calculated. Expressions for them in some detail are given in the following subsections.

\section{B. Equation of state}

The expression for $\ln \mathcal{Z}$ is given by

$$
\begin{aligned}
\ln \mathcal{Z}= & V\left\{\frac{2}{\lambda_{N}^{3}}\left[\zeta_{n}+\zeta_{p}+\frac{b_{n n}}{2} \zeta_{n}^{2}+\frac{b_{p p}}{2} \zeta_{p}^{2}+\frac{1}{2} b_{n p} \zeta_{n} \zeta_{p}\right]\right. \\
& +\frac{2}{\lambda_{t}^{3}}\left[\zeta_{t}+2 \zeta_{t}\left(b_{p t} \zeta_{p}+b_{n t} \zeta_{n}\right)\right] \\
& +\frac{2}{\lambda_{h}^{3}}\left[\zeta_{h}+2 \zeta_{h}\left(b_{p h} \zeta_{p}+b_{n h} \zeta_{n}\right)\right] \\
& \left.+\frac{1}{\lambda_{\alpha}^{3}}\left[\zeta_{\alpha}+b_{\alpha \alpha} \zeta_{\alpha}^{2}+b_{\alpha n} \zeta_{\alpha}\left(\zeta_{n}+\zeta_{p}\right)\right]\right\}+\ln \mathcal{Z}_{\text {Rest }} .
\end{aligned}
$$


In Eq. (19), the subscripts $N, t, h$, and $\alpha$ refer to the nucleon, triton, $\mathrm{He}^{3}$, and $\mathrm{He}^{4}$, respectively. The coefficients $b$ are the virial coefficients. In the limit of isospin symmetry, we take $b_{n n}=b_{p p}$. Due to lack of $p t$ scattering data, we assume $b_{p t} \simeq b_{n h}$. These virial coefficients can be written in terms of experimentally known phase shifts. As an example, we write below explicit expressions for $b_{n n}$ and $b_{n p}$ :

$$
b_{n n}=-\frac{1}{2^{3 / 2}}+\frac{\sqrt{2}}{\pi T} \int_{0}^{\infty} d E \delta_{n n}^{\mathrm{tot}}(E) e^{-\beta E / 2},
$$

and

$$
b_{n p}=b_{n p}^{0}+b_{d}
$$

with

$$
b_{d}=6 \sqrt{2} e^{B_{d} / T}
$$

and

$$
b_{n p}^{0}=-6 \sqrt{2}+\frac{\sqrt{2}}{\pi T} \int_{0}^{\infty} \delta_{n p}^{\mathrm{tot}}(E) e^{-\beta E / 2} .
$$

In Eq. (21), the term $b_{n p}^{0}$ corresponds to the non-resonance $n-p$ scattering contribution; the term $b_{d}$ corresponds to the resonance contribution coming from the bound state of the deuteron with binding energy $B_{d}$. The energy $E$ is measured in the laboratory frame. The expression for the total phase shift is given as

$$
\delta_{N N}^{\text {tot }}=\sum_{L S J}(2 J+1)\left\{\delta_{L_{J}}^{2 S+1}(I=0)+\delta_{L_{J}}^{2 S+1}(I=1)\right\} .
$$

The contributing partial waves are determined by the isospin $I$ with the requirement of the antisymmetry on the total wave function of the $N N$ system.

The last term in Eq. (19) is the sum of the contributions from the rest of the species $(A>4)$ and is given by

$$
\ln \mathcal{Z}_{\text {Rest }}=V \sum_{i} \frac{\zeta_{i}}{\lambda_{i}^{3}}\left(g_{0}+\int_{E_{0}}^{E_{r}} \omega\left(E^{*}\right) e^{-E^{*} / T} d E^{*}\right)
$$

As already stated, the second term in Eq. (25) contributes only for $A>8$. The pressure can be evaluated from

$$
P=T \ln \mathcal{Z} / V
$$

The number density $\rho_{i}$ of the $i$ th fragment species is calculated from

$$
\rho_{i}=\zeta_{i}\left(\frac{\partial}{\partial \zeta_{i}} \frac{\ln \mathcal{Z}}{V}\right)_{V, T}
$$

The total neutron, proton, and baryon density in the system can be obtained from

$$
\begin{aligned}
& \rho_{n}^{B}=\sum_{i} N_{i} \rho_{i}, \\
& \rho_{p}^{B}=\sum_{i} Z_{i} \rho_{i}, \\
& \rho^{B}=\sum_{i} A_{i} \rho_{i} .
\end{aligned}
$$

From the Gibbs-Duhem relation, the free energy density is

$$
\mathcal{F}=-P+\sum_{i} \mu_{i} \rho_{i}
$$

The entropy density $\mathcal{S}$ is calculated from

$$
\mathcal{S}=\left(\frac{\partial P}{\partial T}\right)_{\mu}
$$

which then yields the total energy density as

$$
\mathcal{E}_{\text {tot }}=\mathcal{F}+T \mathcal{S} \text {. }
$$

The detailed expression for the energy density is

$$
\begin{aligned}
\mathcal{E}_{\mathrm{tot}}= & \frac{3}{2} T \sum_{i} \rho_{i}+\sum_{i \in H} \frac{\zeta_{i}}{\lambda_{i}^{3}} \int_{E_{0}}^{E_{r}} \omega\left(E^{*}\right) E^{*} e^{-E^{*} / T} d E^{*} \\
& -\sum_{i} \rho_{i} B_{i}-\frac{3}{2} T\left\{\frac{1}{\lambda_{N}^{3}}\left(b_{n n} \zeta_{n}^{2}+b_{n n} \zeta_{p}^{2}+b_{n p}^{0} \zeta_{n} \zeta_{p}\right)\right. \\
& +\frac{4}{\lambda_{t}^{3}} \zeta_{t}\left(b_{n t} \zeta_{n}+b_{p t} \zeta_{p}\right)+\frac{4}{\lambda_{h}^{3}} \zeta_{h}\left(b_{n h} \zeta_{n}+b_{p h} \zeta_{p}\right) \\
& \left.+\frac{1}{\lambda_{\alpha}^{3}}\left[b_{\alpha n} \zeta_{\alpha}\left(\zeta_{n}+\zeta_{p}\right)+b_{\alpha \alpha} \zeta_{\alpha}^{2}\right]\right\}+\frac{T^{2}}{\lambda_{N}^{3}}\left\{b_{n p}^{0 \prime} \zeta_{n} \zeta_{p}\right. \\
& \left.+b_{n n}^{\prime}\left(\zeta_{n}^{2}+\zeta_{p}^{2}\right)\right\}+\frac{4 T^{2}}{\lambda_{t}^{3}} \zeta_{t}\left(b_{n t}^{\prime} \zeta_{n}+b_{p t}^{\prime} \zeta_{p}\right) \\
& +\frac{4 T^{2}}{\lambda_{h}^{3}} \zeta_{h}\left(b_{n h}^{\prime} \zeta_{n}+b_{p h}^{\prime} \zeta_{p}\right) \\
& +\frac{T^{2}}{\lambda_{\alpha}^{3}}\left\{b_{\alpha \alpha}^{\prime} \zeta_{\alpha}^{2}+b_{\alpha n}^{\prime} \zeta_{\alpha}\left(\zeta_{n}+\zeta_{p}\right)\right\}
\end{aligned}
$$

where $\sum_{i \in H}$ denotes that the sum runs over the channels of heavy particles. In Eq. (32), the first term is identified with $\mathcal{E}_{\text {c.m. }}$, the kinetic energy density associated with the center of mass of the fragments. The second term refers to $\mathcal{E}^{*}$, the sum of the densities of the thermal and resonance excitation energies of the fragments, and the third term coming from the negative of the sum of the fragment binding energies $B_{i}$ is denoted as $\mathcal{E}_{B E}$. The rest is designated as $\mathcal{E}_{I}^{L L}$, the sum of the contributions coming from the interactions between different pairs of light fragments. The primes on the virial coefficients denote their temperature derivatives. They are part of the entropy contributions. Thus, Eq. (32) can be rewritten as

$$
\mathcal{E}_{\mathrm{tot}}=\mathcal{E}_{\mathrm{c} . \mathrm{m} .}+\mathcal{E}^{*}+\mathcal{E}_{B E}+\mathcal{E}_{I}^{L L} .
$$

\section{The single-particle and isovector potentials and the symmetry coefficients}

The single-particle potentials for neutrons or protons in a nuclear medium are conventionally defined as [35]

$$
V_{\tau}=V_{0}+\tau V_{1} X
$$

Here, $V_{0}=\left(V_{n}+V_{p}\right) / 2$ is the isoscalar potential, $V_{1}$ is the measure of the isovector potential, and $\tau= \pm \frac{1}{2}$ for neutrons or protons. The quantity $X=\left(\rho_{n}^{B}-\rho_{p}^{B}\right) / \rho^{B}$ is the asymmetry 
parameter, with $\rho_{\tau}^{B}$ as the total neutron or proton number densities in the system, and $\rho^{B}=\rho_{\tau}^{B}+\rho_{-\tau}^{B}$ is the total nucleon density. The isovector potential $V_{\text {isov }}$ is then

$$
V_{\text {isov }}=V_{n}-V_{p}=V_{1} X \text {. }
$$

In the nucleonic medium, the single-particle potentials are obtained as

$$
V_{\tau}=\left(\frac{\partial \mathcal{E}_{I}}{\partial \rho_{\tau}^{B}}\right)_{\rho_{-\tau}^{B}},
$$

where $\mathcal{E}_{I}$ is the interaction energy density. In homogeneous nuclear matter, it is calculated from the effective interaction. In clusterized nuclear matter, we employ Eq. (36) for the definition of the effective single-nucleon potential. The calculation of $\mathcal{E}_{I}$ is, however, not straightforward. We have adopted the following procedure to take counts of the contributions to the interaction energy density.

In Eq. (33), the first term is purely kinetic and the last term comes solely from the interactions. The binding and the excitation energies, however, are admixtures of both kinetic and interaction contributions. The binding term $\mathcal{E}_{B E}$ can be split as

$$
\mathcal{E}_{B E}=-\sum_{i} \rho_{i} B_{i}=-\sum_{i} \rho_{i}\left(B_{i}^{K}+B_{i}^{I}\right) .
$$

In Eq. (37), the two terms are the kinetic and the interaction parts of the binding energy in a fragment, respectively. The kinetic terms are estimated with the choice of the extended Húlthane wave function for the deuteron [38] and a Gaussian for $t, \mathrm{He}^{3}$, and $\alpha$ particles [39]. The kinetic terms for these light nuclei are very small compared to the total binding term of the system, hence, even a change by $50 \%$ in the estimated values of the kinetic terms in the light nuclei does not change the values of the calculated observables noticeably. For heavier systems, the Fermi gas approximation is employed. Since the binding energies are known experimentally, the interaction contribution $B_{i}^{I}$ can then be obtained. Estimation of the interaction contribution $\mathcal{E}_{I}^{*}$ from the excitation part $\mathcal{E}^{*}$ is not easy. However, compared to the binding term $\sum_{i} \rho_{i} B_{i}^{I}$, it is quite small. It is checked that changes in the single-particle and isovector potentials are insignificant with $\mathcal{E}_{I}^{*}=0$ or $\mathcal{E}_{I}^{*}=\mathcal{E}^{*}$. The calculations reported correspond to $\mathcal{E}_{I}^{*}=\frac{1}{2} \mathcal{E}^{*}$. The interaction energy density then takes the form

$$
\mathcal{E}_{I}=\frac{1}{2} \mathcal{E}^{*}-\sum_{i} \rho_{i}\left(B_{i}-B_{i}^{K}\right)+\mathcal{E}_{I}^{L L},
$$

where we recall that $\mathcal{E}_{I}^{L L}$ refers to the contribution from the interactions between different pairs of light fragments. Using Eqs. (27), (36), and (38), the effective single-particle and isovector potentials in clusterized matter can then be calculated. The single-particle potentials are called effective in the sense that they represent the average of the interaction of a single nucleon with all other free nucleons and bound nucleons in clusters of different sizes, the clusters being present in different proportions in the inhomogeneous system.

The total symmetry energy coefficient $C_{s}^{\text {tot }}$ is defined through

$$
e(X)=e(X=0)+C_{s}^{\text {tot }} X^{2},
$$

where $e=\mathcal{E}_{\text {tot }} / \rho^{B}$ is the energy per nucleon. The coefficient $C_{s}^{\text {tot }}$ can be split into interaction and kinetic terms as

$$
C_{s}^{\mathrm{tot}}=C_{s}^{I}+C_{s}^{K},
$$

corresponding to

$$
\begin{gathered}
e_{I}(X)=e_{I}(X=0)+C_{s}^{I} X^{2}, \\
e_{K}(X)=e_{K}(X=0)+C_{s}^{K} X^{2} .
\end{gathered}
$$

In nuclear matter, the isovector potential is related to the interaction energy per nucleon $e_{I}(X)$ as $V_{\text {isov }}=2\left(\partial e_{I}(X) / \partial X\right)_{\rho^{B}}$. For homogeneous matter, $e_{I}(X)$ is linear in $X^{2}$, then

$$
V_{\mathrm{isov}}=4 C_{s}^{I} X \text {. }
$$

For clusterized matter, this relation is, however, only approximate because $e_{I}(X)$ (as well as the energy per nucleon $e(X)[31])$ is seen to be not fully linear in $X^{2}$.

The incompressibility $K(X)$ of a nuclear system is calculated from

$$
K(X)=9 \frac{d P}{d \rho^{B}},
$$

where $P$, given by Eq. (26), is the pressure of the system with asymmetry $X$. It can be written as

$$
K(X)=K(X=0)+K_{s} X^{2},
$$

where the coefficient $K_{s}$ is the symmetry incompressibility.

\section{RESULTS AND DISCUSSIONS}

We have calculated the equation of state (EOS), the single-particle and isovector potentials, symmetry energy coefficients and the symmetry incompressibility of dilute nuclear and supernova matter at different temperatures and densities. Results for nuclear matter are presented in Sec. III A and those for supernova matter are given in Sec. III B. Calculations have been restricted up to a baryon density $\rho^{B}=0.02 \mathrm{fm}^{-3}$. At relatively high density, the asymptotic wave function may not have a precise meaning and then expressions of the partition function in terms of $S$-matrix elements may not be very meaningful.

The virial coefficients related to $N N, N \alpha$, and $\alpha \alpha$ scatterings along with their temperature derivatives are taken from Ref. [32]. The same for $N t$ and $N h$ scatterings are obtained from Ref. [40]. In doing so, appropriate care has been taken for the slightly different choices of factors in the virial expansion of the partition function.

\section{A. Nuclear matter}

In Fig. 1, the pressure $P$ is displayed as function of baryon density for symmetric $(X=0.0)$ and asymmetric $(X=0.3)$ nuclear matter. The upper panel corresponds to a temperature $T=4 \mathrm{MeV}$, the lower panel corresponds to $T=8 \mathrm{MeV}$. The results calculated in the $S$-matrix approach (SM) are compared with those in a mean-field (MF) model. The MF calculations are performed with the $\mathrm{SkM}^{*}$ interaction. At very low densities, $P$ is nearly model independent $(P \simeq \rho T)$; at a little higher density, the difference between the two models is apparent from the figure. In the MF calculation, 


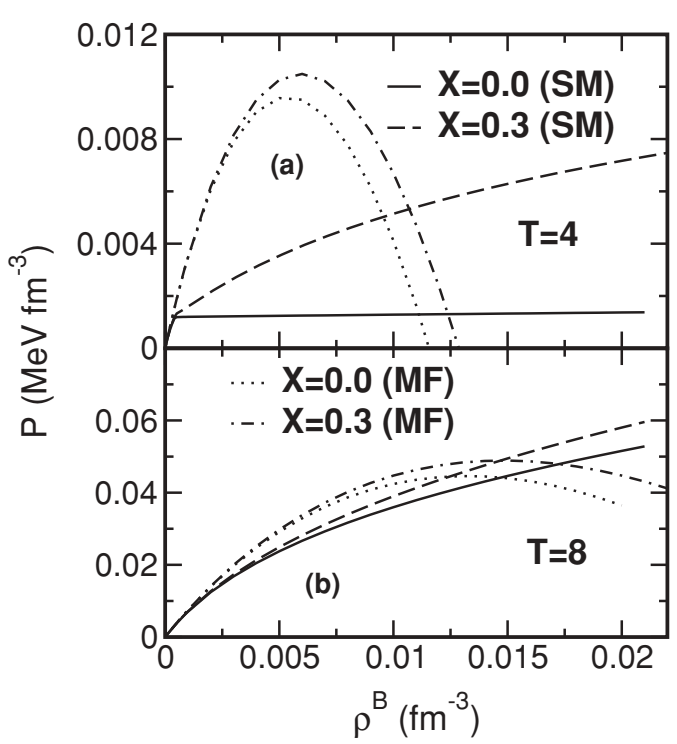

FIG. 1. The isotherms for nuclear matter at $T=4 \mathrm{MeV}$ (upper panel) and $T=8 \mathrm{MeV}$ (lower panel) for symmetric $(X=0.0)$ and asymmetric ( $X=0.3$ ) nuclear matter in the $S$-matrix (SM) and meanfield (MF) models.

with isothermal compression, the dilute system enters the unphysical region beyond a certain density which increases with temperature. In the SM approach, with compression, the unphysical behavior does not arise because of many-body correlations (condensation). For symmetric matter, at low temperature, the pressure levels off at very low densities as shown by the full line in the upper panel signaling a behavior like a first-order phase transition. It points out a phase coexistence between light and heavy clusters. For asymmetric matter, the rise in pressure is linear in density at constant temperature for very dilute systems. There is a break from this linearity at a certain density (we refer to this density as the condensation density); the pressure thereafter rises monotonically behaving like a second-order phase transition. At the higher temperature $T=8 \mathrm{MeV}$, the said transition occurs at a much higher density which is beyond the density we consider. The increase in free neutron multiplicity with density is mainly responsible for this monotonic rise. For both symmetric and asymmetric systems, the chemical equilibrium conditions coupled with the conservation of the baryon number and isospin governs this behavior.

Since there is no Coulomb in the binding energy of the fragments formed in nuclear matter, the sum in Eq. (3) runs up to infinity in principle; in practice, one takes a finite sum for calculational facilitation. The calculations here have been performed with a maximum fragment mass $A_{\max }=1000$. The results are not very sensitive to further increase in the maximum mass [31]. The binding energies of these nuclei are obtained from the liquid-drop type mass formula [41] with Coulomb switched off.

The effective single-particle potentials $V_{n}$ and $V_{p}$ for neutron and proton are shown in Fig. 2 as a function of density at temperatures $T=4$ and $8 \mathrm{MeV}$ for symmetric and asymmetric $(X=0.3)$ nuclear matter. The isovector potential $V_{n}-V_{p}$ is also shown in the bottom panels for

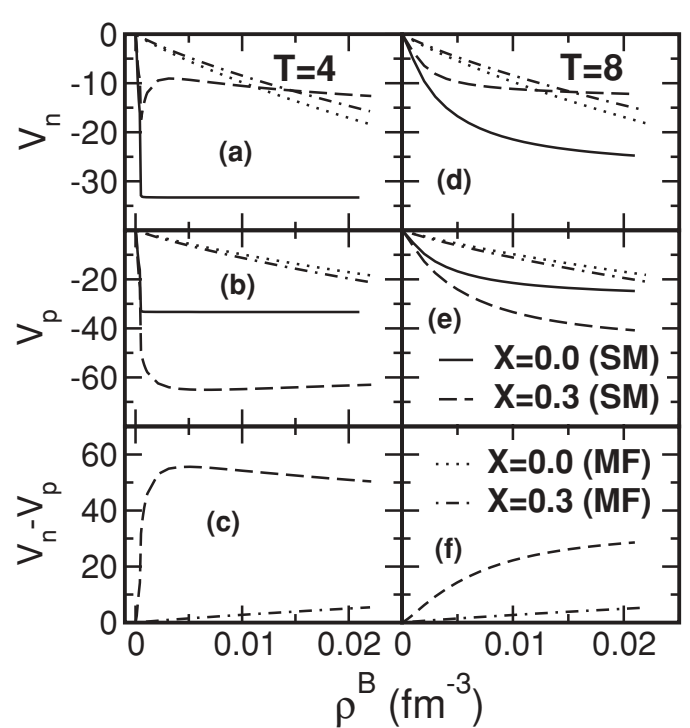

FIG. 2. The single-nucleon $\left(V_{n}\right.$ and $\left.V_{p}\right)$ and isovector $\left(V_{n}-V_{p}\right)$ potentials in $\mathrm{MeV}$ shown at $T=4 \mathrm{MeV}$ (left panels) and $T=8 \mathrm{MeV}$ (right panels) for symmetric $(X=0.0)$ and asymmetric $(X=0.3)$ nuclear matter in the SM and MF models as a function of the baryon density.

asymmetric matter at the same temperatures. Calculations have been performed in both the SM and MF approaches. In the mean-field model, the single-nucleon potentials decrease with increasing density with a monotonic increase of the isovector potential. They are nearly independent of temperature. In the $S$-matrix approach, the single-nucleon potentials show a subtle behavior with density. For symmetric matter, $V_{n}\left(=V_{p}\right)$, at lower temperature decreases sharply up to the condensation density, beyond which it remains practically constant. Beyond this density, the fragment composition of matter scales nearly with density and the constancy of the single-particle potential is a reflection of that. The value of this constant effective single-particle potential is $\sim-34 \mathrm{MeV}$ in contrast to that of $\sim-60 \mathrm{MeV}$ for saturated uniform nuclear matter. This is so because in calculating $\delta \mathcal{E}_{I} / \delta \rho$ for uniform matter, one has to count the change in nucleon density, but in clusterized matter, the internal nucleonic density of fragments remains unaltered, only their number density changes. At lower temperature, for asymmetric nuclear matter, $V_{n}$ goes through a sharp minimum around the condensation density. Beyond this density, it passes through a maximum and then decreases slowly; $V_{p}$ on the other hand behaves more like that of symmetric nuclear matter, but it is deeper. The above qualitatively different behavior of $V_{n}$ and $V_{p}$ comes from the presence of free neutrons in asymmetric nuclear matter. The isovector potential passes through a maximum and then decreases slowly with density. As in mean-field, the isovector potential is seen to be nearly proportional to the asymmetry $X$. The different nature of the single-particle potentials at higher temperature as seen for $T=8 \mathrm{MeV}$ is a manifestation of the dilution of condensation effects.

The symmetry energy coefficients, as a function of baryon density, are displayed in the left and right panels of Fig. 3 at temperatures $T=4$ and $8 \mathrm{MeV}$, respectively. In the MF 


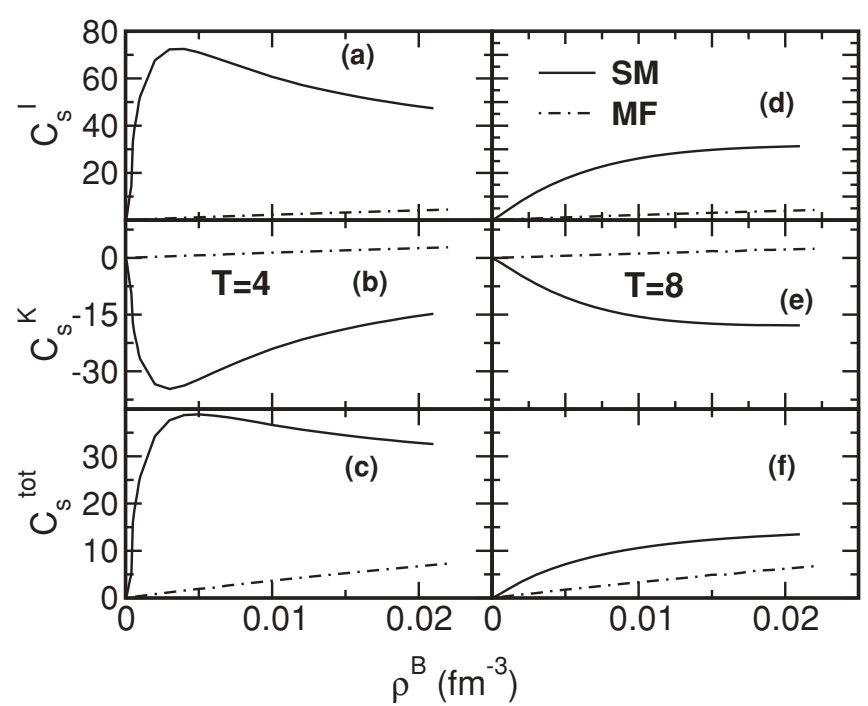

FIG. 3. The interaction, kinetic, and total symmetry energy coefficients $\left(C_{s}^{I}, C_{s}^{K}\right.$, and $C_{s}^{\text {tot }}$, respectively, all in $\left.\mathrm{MeV}\right)$ for nuclear matter shown as a function of the baryon density at $T=4 \mathrm{MeV}$ (left panels) and $T=8 \mathrm{MeV}$ (right panels) in the SM and MF models.

approach, the total symmetry coefficient $C_{s}^{\text {tot }}$ along with the kinetic and interaction components $C_{s}^{K}$ and $C_{s}^{I}$ (shown as dot-dash lines) increase linearly with density and are seen to be practically independent of temperature. The symmetry energy $e_{\text {sym }}(X)=e(X)-e(X=0)$ in the SM approach, as opposed to that in the MF model, is found to be anharmonic in $X$, particularly at lower temperatures; this was already noted earlier [31]. This induces an asymmetry dependence in the symmetry coefficients defined through Eqs. (39) and (41). To avoid this, we therefore, take the definition [32]

$$
C_{s}=\frac{1}{2}\left(\frac{\partial^{2} e(X)}{\partial X^{2}}\right)_{X=0} .
$$

This applies to $C_{s}^{I}, C_{s}^{K}$, and $C_{s}^{\text {tot }}$ with appropriate choice of the energy components. The symmetry coefficients, so defined are shown as full lines in the figure. The symmetry coefficients thus obtained are seen to be very different from those obtained in the MF model. The magnitudes of the coefficients are much larger, the kinetic component is always negative and there is a marked dependence on temperature. The negative symmetry kinetic energy $C_{s}^{K} X^{2}\left(=e_{K}(X)-e_{K}(X=0)\right)$ looks anti-intuitive. For clusterized nuclear matter, it, however, can be understood from the fact that for symmetric matter, clusterization is favored leading to larger internal kinetic energy compared to asymmetric matter where there are more free neutrons and have lesser total internal kinetic energy.

The anharmonicity of the symmetry energy $e_{\text {sym }}(X)$ [calculated in the SM approach through Eq. (32)] in the asymmetry parameter is portrayed in Fig. 4. In the figure the evolution of $D_{S E}$, a measure of this anharmonicity, is shown as a function of baryon density $\rho^{B}$ at different $X$ at temperatures $T=4$ (full line) and $8 \mathrm{MeV}$ (dashed line), respectively. The anharmonicity parameter $D_{S E}$ is defined as

$$
D_{S E}=\frac{C_{s}^{\mathrm{tot}} X^{2}-e_{\mathrm{sym}}(X)}{C_{S}^{\mathrm{tot}} X^{2}} \times 100 .
$$

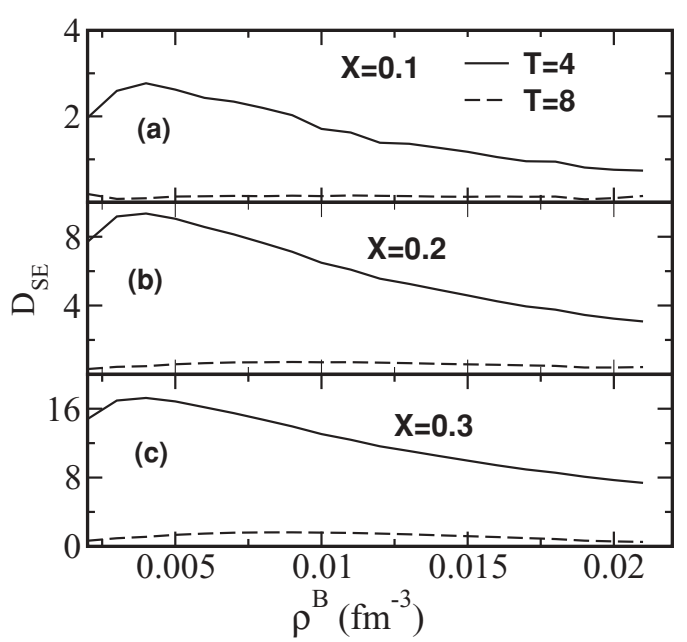

FIG. 4. The anharmonicity $D_{S E}$ of the symmetry energy (see text) plotted as a function of the baryon density at $T=4$ and $8 \mathrm{MeV}$ for three different asymmetries.

It represents the percentage deviation of $e_{\text {sym }}(X)$ from $C_{s}^{\text {tot }} X^{2}$ where $C_{s}^{\text {tot }}$ is defined through Eq. (45). As expected, anharmonicity increases with asymmetry. On the other hand, it decreases with increasing temperature because of the dissolution of clusters with heating.

In Fig. 5, the incompressibility coefficient $K$ of symmetric $(X=0.0)$ and asymmetric $(X=0.3)$ nuclear matter are presented in the left panels as a function of density at $T=4$ and $8 \mathrm{MeV}$. At very low density, the incompressibility in both the SM and MF approaches is nearly the same. Increasing density and subsequent condensation renders the system more compressible in the SM approach. The somewhat different behavior of the incompressibility for symmetric and asymmetric matter at lower temperature in the $S$-matrix approach can be easily understood from the different functional dependence of

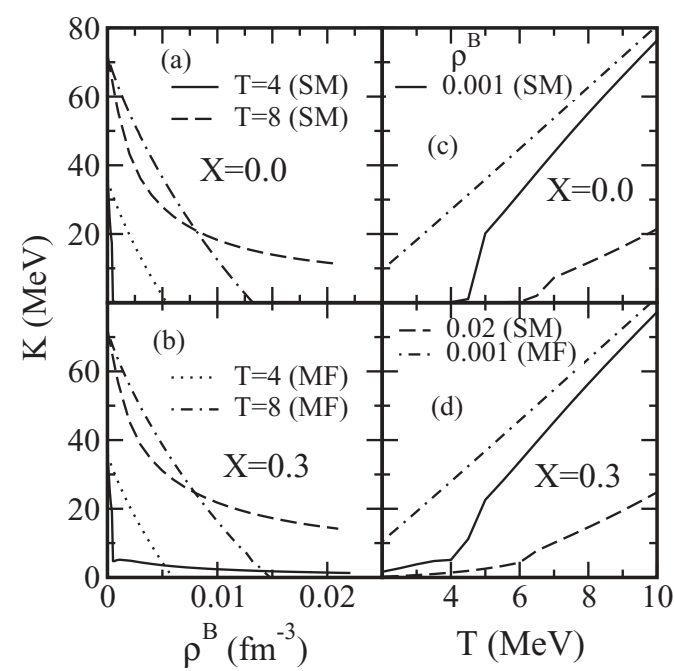

FIG. 5. The incompressibility coefficient $K$ shown as a function of the baryon density at $T=4$ and $8 \mathrm{MeV}$ for symmetric and asymmetric nuclear matter (left panels) in the SM and MF models. In the right panels $K$ is shown as a function of temperature in the two models at the baryon densities indicated. 


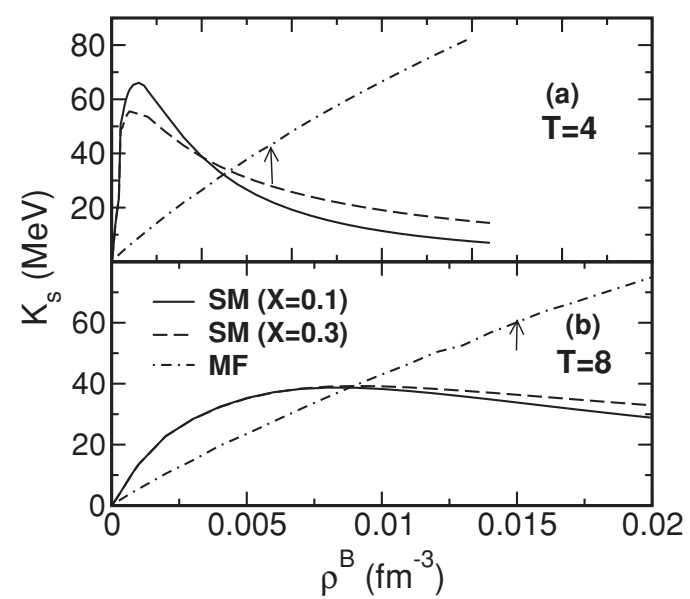

FIG. 6. The symmetry incompressibility coefficient $K_{s}$ shown as a function of the baryon density at $T=4 \mathrm{MeV}$ (upper panel) and $T=8 \mathrm{MeV}$ (lower panel) for asymmetric nuclear matter with $X=0.1$ and 0.3 in the SM and MF models. In the MF model, $K_{s}$ is independent of asymmetry. The arrows denote the density beyond which the system enters the unphysical region in the MF model.

pressure with density for symmetric and asymmetric systems as shown in Fig. 1. In the MF approach, on isothermal compression, the system enters the unphysical region beyond a certain density where the incompressibility becomes negative. This is not shown in the figure. From the right panels of this figure, it is seen that in the physical region, at a fixed density, the incompressibility $K$ decreases almost linearly on isochoric cooling. In the SM approach, on isochoric cooling, there is a linear decrease in $K$, however, a sudden fall in incompressibility at a certain temperature is noticed. This is related to the onset of condensation where the fragment multiplicity suddenly drops [42].

The symmetry incompressibility $K_{s}$ defined in Eq. (44) is compared in the SM and MF models in Fig. 6 at temperatures $T=4$ and $8 \mathrm{MeV}$. As opposed to the MF model where $K_{s}$ increases monotonically in the density region we explore, the same in the SM approach has a peaked structure which is prominent at lower temperature. Here, the symmetry incompressibility is seen to be weakly dependent on the asymmetry of the system while in the MF model it is asymmetry independent. The arrows in the figure indicate the density above which the system enters the unphysical region in the mean-field model.

In Fig. 7, the symmetry incompressibility $K_{S}$ is shown as a function of temperature at baryon densities $\rho^{B}=0.001$ and $0.01 \mathrm{fm}^{-3}$. In contrast to the mean-field results where $K_{s}$ is practically independent of temperature, in the $S$-matrix approach, $K_{s}$ is sharply peaked around the condensation region. The asymmetry dependence of $K_{s}$ in this approach washes out with increasing temperature. The arrow in the lower panel of this figure marks the temperature below which the system is in the unphysical region in the MF model.

\section{B. Supernova matter}

Neutrons, protons, and electrons are assumed to be the basic constituents of the supernova matter we are dealing with. The

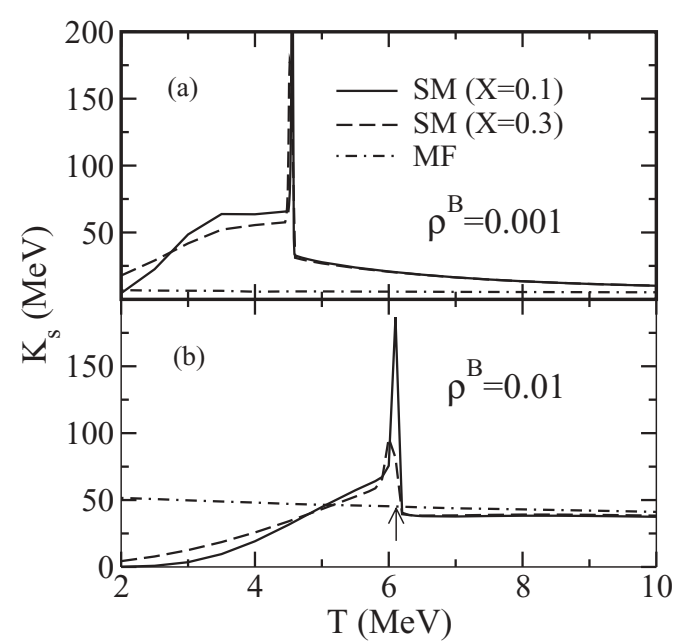

FIG. 7. The symmetry incompressibility $K_{s}$ for asymmetric nuclear matter shown as a function of temperature at baryon densities $\rho^{B}=0.001$ and $0.01 \mathrm{fm}^{-3}$ in the SM and MF models. In the MF model, $K_{s}$ does not depend on asymmetry. The arrow in panel (b) denotes the temperature below which the system enters the unphysical region in the MF model.

matter is also assumed to be in $\beta$ equilibrium. For charge neutrality, the electron number density $\rho_{e}=\rho_{p}^{B}$, the proton number density. This $\beta$-equilibrated matter is treated both in the mean-field (MFB) and the $S$-matrix (SMB) approaches. The proton fraction in the system depends on its baryon density and temperature. In supernova matter, in the SMB approach, the fragments have Coulomb contribution in the binding energy; so, in the sum in Eq. (3), only nuclei within the drip lines are considered. For this purpose, all the isotopes (around 9000 in number) and their binding energies are taken from Ref. [43]. The effect of electron environment is taken into account through the dressing up of the fragment binding energies in the Wigner-Seitz approximation [34,44]. For a given electron density $\rho_{e}$, the effective binding energy of the $i$ th fragment species with $A_{i}$ and $Z_{i}$ as its mass and atomic number is given by

$$
B_{i}\left(\rho_{e}\right)=B_{i}(0)+\Delta B_{i},
$$

where

$$
\Delta B_{i}=\frac{3}{5} \frac{Z_{i}^{2} e^{2}}{R_{0 i}}\left(\frac{3}{2} \eta_{i}-\frac{1}{2} \eta_{i}^{3}\right)
$$

with

$$
\eta_{i}=\left(\frac{\rho_{e}}{\rho_{0}} \frac{A_{i}}{Z_{i}}\right)^{1 / 3}
$$

In the above equations, $\rho_{0}$ is the saturation density of normal nuclear matter, $R_{0 i}=1.16 A_{i}^{1 / 3} \mathrm{fm}$ is the radius of the fragment of mass $A_{i}$ and charge $Z_{i}$. For proton, $R_{0 i}$ is taken as $0.8 \mathrm{fm}$. The increase in the effective binding energy acts to enhance the formation of heavier nuclei.

The electron fractions $Y_{e}\left(=\rho_{e} / \rho^{B}\right)$ for the supernova matter are compared in the SMB and MFB models as a function of baryon density $\rho^{B}$ at temperature $T=4$ and $8 \mathrm{MeV}$ in Fig. 8. The two results follow nearly the same trend 


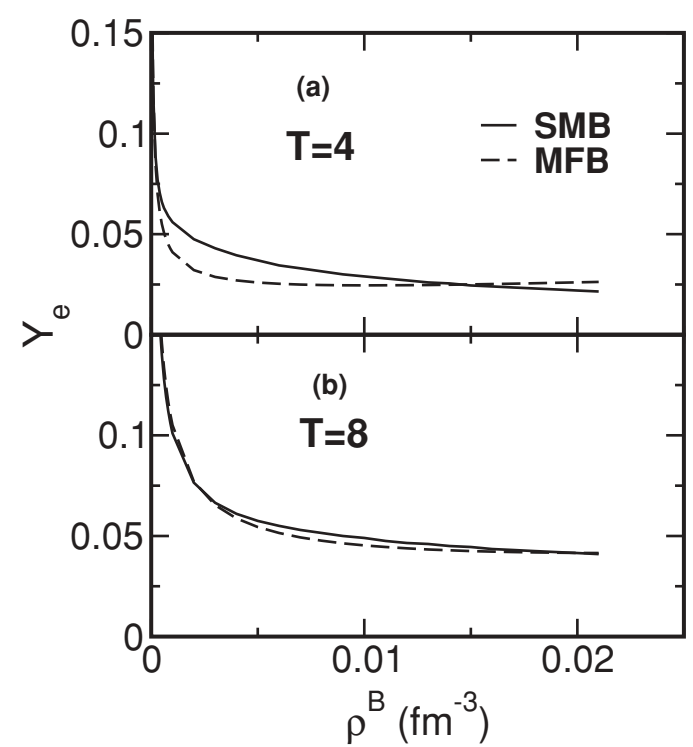

FIG. 8. The electron fraction $Y_{e}$ in $\beta$-equilibrated supernova matter as a function of the baryon density at $T=4$ and $8 \mathrm{MeV}$ in the $S$-matrix (SMB) and mean-field (MFB) models.

(increasingly neutron-rich with increasing baryon density); the SMB model, however, predicts somewhat less neutron-rich system indicating an effectively lower $e^{-}$-capture rate which may be significant enough to influence supernova dynamics [1], particularly at lower temperature. In Fig. 9, the calculated baryonic pressure from the two models at the aforesaid temperatures are shown as a function of density. In contrast to nuclear matter, the baryonic pressure for supernova matter in the two models are seen to be nearly the same. This is due to the fact that in supernova matter, to maintain $\beta$ equilibrium, the asymmetry is very high, so much of the matter is in the free neutron state that effectively controls the behavior of the pressure.

The variation of the symmetry energy coefficients $\left(C_{s}^{I}, C_{s}^{K}\right.$, and $C_{s}^{\text {tot }}$ ) of supernova matter as a function of density at temperatures $T=4$ and $8 \mathrm{MeV}$ are shown in Fig. 10. These

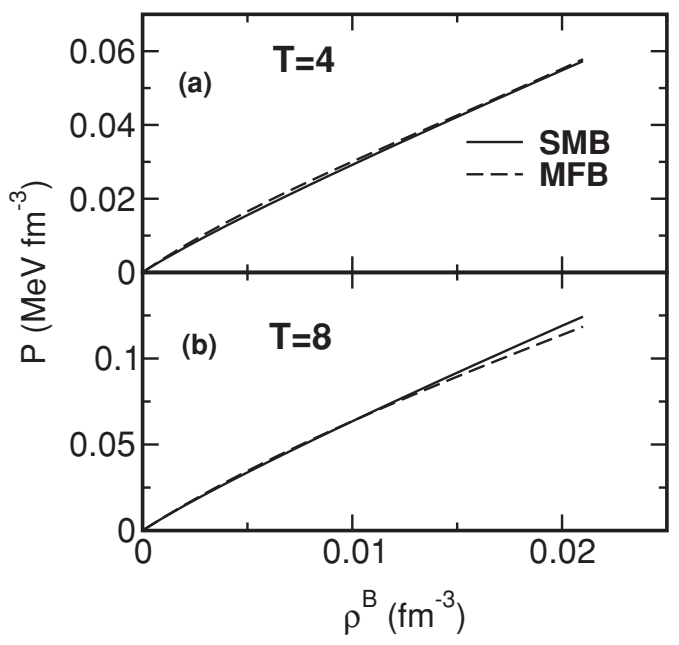

FIG. 9. The isotherms for $\beta$-equilibrated supernova matter at $T=4$ and $8 \mathrm{MeV}$ in the SMB and MFB models.

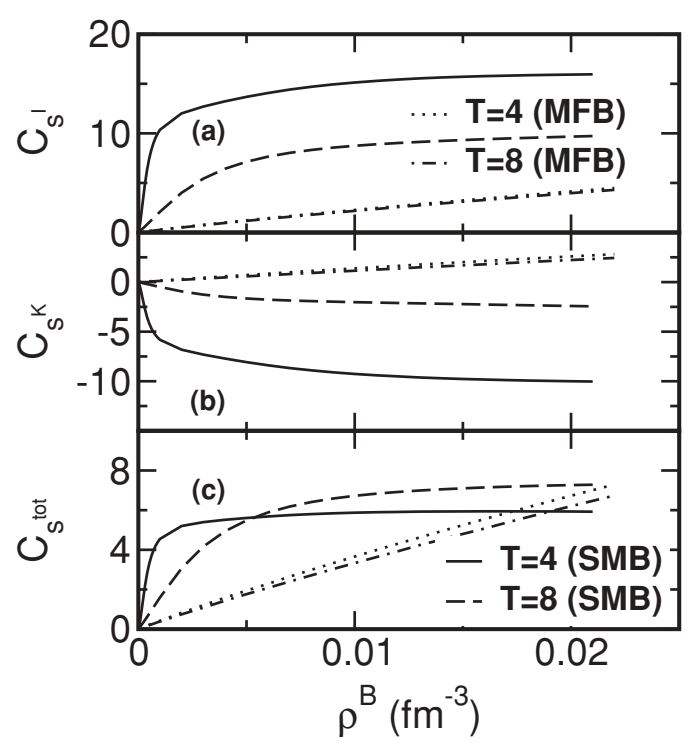

FIG. 10. The symmetry energy coefficients $C_{s}^{I}, C_{s}^{K}$, and $C_{s}^{\text {tot }}$ (in $\mathrm{MeV}$ ) of $\beta$-equilibrated supernova matter as a function of the baryon density for $T=4$ and $8 \mathrm{MeV}$ in the SMB and MFB models.

coefficients are evaluated using Eqs. (39) and (41) at the relevant asymmetries. The MFB results are the same as those of nuclear matter as shown in Fig. 3 as they are asymmetry independent. In the $S$-matrix approach to supernova matter, the symmetry energy coefficients have the same trend with density as in nuclear matter, however, the values get much reduced. The presence of Coulomb in the fragment binding energy hinders the formation of heavier fragments and hence this reduction.

The baryonic incompressibility of supernova matter is shown in Fig. 11 as a function of the baryonic density in the SMB and MFB models at $T=4$ and $8 \mathrm{MeV}$. The results in the two models are qualitatively the same at both the

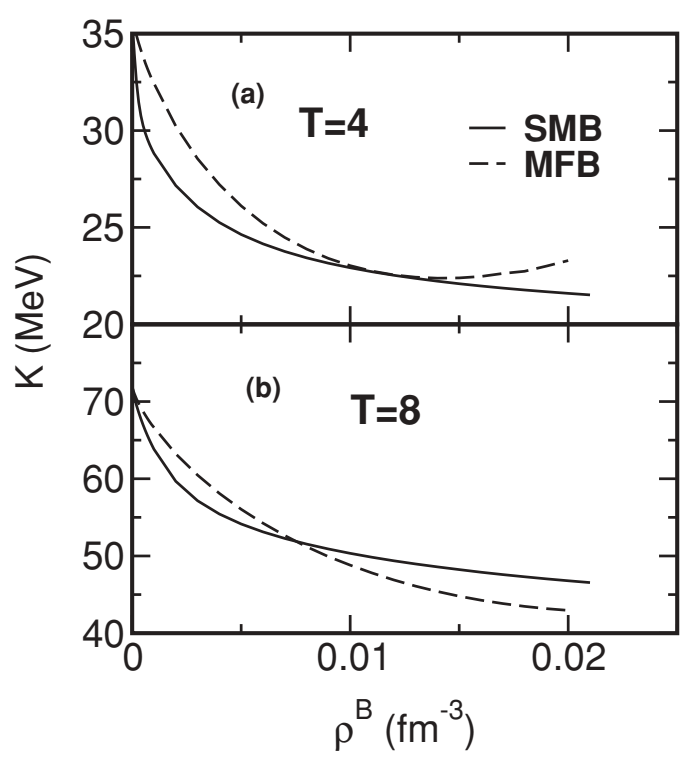

FIG. 11. The incompressibility of $\beta$-equilibrated supernova matter as a function of the baryon density at $T=4$ and $8 \mathrm{MeV}$ in the SMB and MFB models. 


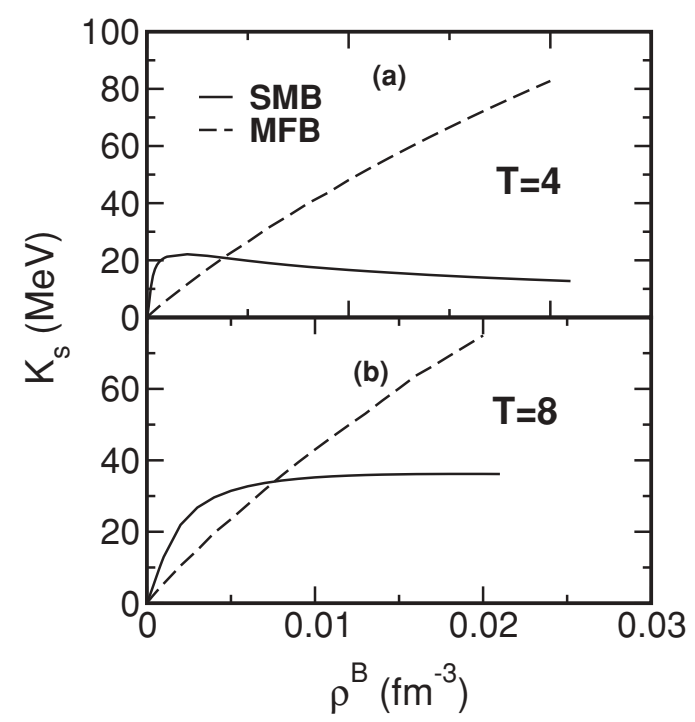

FIG. 12. The symmetry incompressibility $K_{s}$ for supernova matter as a function of the baryon density at $T=4$ and $8 \mathrm{MeV}$ in the SMB and MFB models.

temperatures and are different from those obtained for nuclear matter shown in Fig. 5. The calculated incompressibility for supernova matter is seen to be significantly larger to that in nuclear matter except at very low density where both nuclear and supernova matter are mostly composed of free nucleons.

The baryonic symmetry incompressibilities $K_{s}$ for supernova matter in the two models at the aforesaid temperatures are displayed in Fig. 12. In the mean-field model, $K_{s}$ is independent of asymmetry, hence the MFB results are the same as the MF results as shown in Fig. 6. In the $S$-matrix approach, the symmetry incompressibility for supernova matter at a temperature of $4 \mathrm{MeV}$ is much reduced compared to that in nuclear matter at lower density; at higher temperature, however, the corresponding two results are close due to absence of formation of heavier fragments within the density range studied. The differences between the incompressibilities obtained for nuclear matter and supernova matter arise because calculations in nuclear matter refer to a fixed asymmetry which is taken to be relatively low, but the calculations for supernova matter, with changing density, pertain to varying asymmetries which turn out to be quite high. Furthermore, the presence of the Coulomb contribution in the fragment binding energies precludes the formation of very heavy fragments, thereby increasing the pressure.

\section{CONCLUDING REMARKS}

The low-density nuclear or supernova matter is not homogeneous, it is clusterized at a finite temperature. In this paper, we have investigated some structural properties of this low density matter in the the $S$-matrix framework. Special emphasis has been given to understand the effects brought out by the isospin structure of the nuclear force. In particular, we have considered its effects on the energy, incompressibility and the singlenucleon potentials. The effects are more clearly manifest in the symmetry energy coefficients, symmetry incompressibility and isovector potential.

In the $S$-matrix approach, symmetric or asymmetric nuclear matter condenses on isothermal compression thereby avoiding the unphysical region brought out in the mean-field model. Condensation eases pressure, the incompressibility is thus generally lower. Condensation has a marked effect on the symmetry incompressibility $K_{s}$. Around the condensation density, $K_{s}$ shows a peaked structure, particularly at lower temperature, in contrast to its smooth monotonic increase with density in the mean-field model. In the supernova matter, the isotherms, so also the incompressibility in the two models are, however, not very different; the rapid neutronization in the $\beta$-equilibrated matter with increasing density is mostly responsible for this change. A remarkable feature of the symmetry energy in the $S$-matrix approach for nuclear matter is that as opposed to results from mean-field, the symmetry energy is nonlinear in $X^{2}$. The symmetry coefficients are also larger in magnitude in the SM model and that the kinetic components are, contrary to expectations, negative. The same trends follow in supernova matter.

In the $S$-matrix framework, the calculated properties like the equation of state, the total symmetry energy coefficient and the incompressibilities of nuclear or supernova matter do not depend on any particular choice of the nuclear force, they are model independent. They can be directly connected to the experimentally measured phase shifts and the binding energies of the fragments constituting the matter. The $e^{-}$capture envisaged through the chemical equilibrium conditions in supernova matter is similarly independent of any model for nuclear interactions. At low temperatures, in a selective density range, the $S$-matrix model predicts a somewhat higher electron fraction in the system compared to the mean-field calculations indicating lower $e^{-}$-capture rate. These may, however, play a significant role in supernova dynamics.

\section{ACKNOWLEDGMENTS}

S.K.S. and J.N.D. acknowledge support of DST, Government of India. M.C. and X.V. were partially supported by the Consolider Ingenio 2010 Programme CPAN CSD2007-00042 and Grants FIS2008-01661 from MEC and FEDER and 2009SGR-1289 from Generalitat de Catalunya.
[1] H. A. Bethe, Rev. Mod. Phys. 62, 801 (1990).

[2] H.-Th. Janka, K. Langanke, A. Marek, G. Martínez-Pinedo, and B. Müller, Phys. Rep. 442, 38 (2007).

[3] A. W. Steiner, M. Prakash, J. M. Lattimer, and P. J. Ellis, Phys. Rep. 411, 325 (2005).

[4] H. Stöcker and W. Greiner, Phys. Rep. 137, 277 (1986).
[5] P. Danielewicz, R. Lacey, and W. G. Lynch, Science 298, 1592 (2002).

[6] V. Baran, M. Colonna, V. Greco, and M. Di Toro, Phys. Rep. 410, 335 (2005).

[7] B. A. Li, L. W. Chen, and C. M. Ko, Phys. Rep. 464, 113 (2008), and references therein. 
[8] G. Ferini, T. Gaitanos, M. Colonna, M. Di Toro, and H. H. Wolter, Phys. Rev. Lett. 97, 202301 (2006).

[9] Z. Xiao, B. A. Li, L. W. Chen, G. C. Yong, and M. Zhang, Phys. Rev. Lett. 102, 062502 (2009).

[10] D. V. Shetty, S. J. Yennello, and G. A. Souliotis, Phys. Rev. C 76, 024606 (2007).

[11] S. Kowalski et al., Phys. Rev. C 75, 014601 (2007).

[12] S. K. Samaddar, J. N. De, X. Viñas, and M. Centelles, Phys. Rev. C 78, 034607 (2008).

[13] A. S. Botvina, N. Buyukcizmeci, M. Erdogan, J. Lukasik, I. N. Mishustin, R. Ogul, and W. Trautmann, Phys. Rev. C 74, 044609 (2006).

[14] M. B. Tsang et al., Phys. Rev. Lett. 92, 062701 (2004).

[15] L. W. Chen, C. M. Ko, and B. A. Li, Phys. Rev. Lett. 94, 032701 (2005).

[16] M. B.Tsang, Y. Zhang, P. Danielewicz, M. Famiano, Z. Li, W. G. Lynch, and A. W. Steiner, Phys. Rev. Lett. 102, 122701 (2009).

[17] G. A. Souliotis, A. S. Botvina, D. V. Shetty, A. L. Keksis, M. Jandel, M Veselsky, and S. J. Yennello, Phys. Rev. C 75, 011601(R) (2007).

[18] K. Sumiyoshi, H. Suzuki, and H. Toki, Astron. Astrophys. 303, 475 (1995).

[19] D. Bandyopadhyay, C. Samanta, S. K. Samaddar, and J. N. De, Nucl. Phys. A511, 1 (1990).

[20] E. Chabanat, P. Bonche, P. Haensel, J. Meyer, and R. Schaeffer, Nucl. Phys. A627, 710 (1997).

[21] J. Piekarewicz, Phys. Rev. C 69, 041301(R) (2004).

[22] G. Colò, N. V. Giai, J. Meyer, K. Bennaceur, and P. Bonche, Phys. Rev. C 70, 024307 (2004).

[23] V. A. Dexheimer, C. A. Z. Vasconcellos, and B. E. J. Bodmann, Phys. Rev. C 77, 065803 (2008)

[24] J. Piekarewicz and M. Centelles, Phys. Rev. C 79, 054311 (2009).
[25] H. Heiselberg and M. Hjorth-Jensen, Phys. Rep. 328, 237 (2000).

[26] R. J. Furnstahl, Nucl. Phys. A706, 85 (2002).

[27] C. Fuchs and H. H. Wolter, Eur. Phys. J. A 30, 5 (2006).

[28] J. Xu, L. W. Chen, B. A. Li, and H. R. Ma, Phys. Rev. C 75, 014607 (2007).

[29] M. Centelles, X. Roca-Maza, X. Viñas, and M. Warda, Phys. Rev. Lett. 102, 122502 (2009).

[30] S. Mallik, J. N. De, S. K. Samaddar, and Sourav Sarkar, Phys. Rev. C 77, 032201(R) (2008).

[31] J. N. De and S. K. Samaddar, Phys. Rev. C 78, 065204 (2008).

[32] C. J. Horowitz and A. Schwenk, Nucl. Phys. A776, 55 (2006).

[33] R. Dashen, S.-k. Ma, and H. J. Bernstein, Phys. Rev. 187, 345 (1969).

[34] S. L. Shapiro and S. A. Teukolsky, Black Holes, White Dwarfs, and Neutron Stars (John Wiley \& Sons. Inc., New York, 1983).

[35] A. Bohr and B. R. Mottelson, Nuclear Structure, Vol. I (W. A. Benjamin Inc., Reading, MA, 1969).

[36] R. Dashen and R. Rajaraman, Phys. Rev. D 10, 694 (1974).

[37] R. Dashen and R. Rajaraman, Phys. Rev. D 10, 708 (1974).

[38] Ronald J. Adler, T. K. Das, and Alvaro Ferraz Filho, Phys. Rev C 16, 1231 (1977).

[39] L. I. Schiff, Phys. Rev. 133, B802 (1964).

[40] E. O'Connor, D. Gazit, C. J. Horowitz, A. Schwenk, and N. Barnea, Phys. Rev. C 75, 055803 (2007).

[41] P. Danielewicz, Nucl. Phys. A727, 233 (2003).

[42] J. N. De and S. K. Samaddar, Phys. Rev. C 76, 044607 (2007).

[43] W. D. Myers and W. J. Swiatecki, Nucl. Phys. A601, 141 (1996); Lawrence Berkeley Laboratory Report LBL-36803.

[44] C. Ishizuka, A. Ohnishi, and K. Sumiyoshi, Nucl. Phys. A723, 517 (2003). 